\title{
ERRATA
}

\section{D SADDLE POTENTIAL SCHRÖDINGER GREEN'S FUNCTION IN THE PRESENCE OF AN ARBITRARY TIME-DEPENDENT ELECTRIC FIELD}

\author{
[Mod. Phys. Lett. B, Vol. 11, Nos. 26 \& 27 (1997) 1193-1196] \\ NORMAN J. M. HORING and KASHIF SABEEH
}

On p. 1193, first line of first paragraph following abstract, misspelled "cinsiderable" should read as "considerable".

Second line of first paragraph following abstract, misspelled "fileds" should read as "fields".

On p. 1193, Eq. (2) should read as follows:

$$
\hat{H}(t)=\frac{\hat{p}_{1}^{2}}{2 m}+\frac{\hat{p}_{2}^{2}}{2 m}+\frac{1}{2} k_{1} \hat{x}_{1}^{2}-\frac{1}{2} k_{2} \hat{x}_{2}^{2}-e \mathbf{E}(t) \cdot \hat{x},
$$

On p. 1194, Eq. (6b) should read as follows:

$$
\frac{d^{2} \hat{x}_{2}}{d t^{2}}-\frac{k_{2}}{m} \hat{x}_{2}=\frac{e}{m} E_{2}(t)
$$

On p. 1196, second last term of Eq. (12) should read as

$$
+\frac{e^{2}}{m \omega_{2} \sinh \omega_{2} t} \int_{0}^{t} d t^{\prime} \int_{0}^{t^{\prime}} d t^{\prime \prime} \tilde{E}_{2}\left(t^{\prime}\right)
$$

